\title{
Avaliação do Conhecimento da Saúde Bucal Entre Pacientes Cardiopatas Antes da Realização de Cirurgia Cardíaca em um Hospital Universitário
}

\section{Assessment of Oral Health Knowledge among Cardiac Patients before Heart Surgery in a School Hospital}

\author{
Antônio Marcos da Silva Nobreza $a^{a}$ Tereza Aparecida Delle Vedove Semenoff $f^{\text {b }}$ Natalino Francisco da Silva ${ }^{\mathrm{b}}$ \\ Veroneide Pio da Silva Leandro ${ }^{\text {cd }}$; Álvaro Henrique Borges ${ }^{\mathrm{b}}$; Alex Semenoff Segundo ${ }^{\mathrm{b} *}$ \\ ${ }^{a}$ Universidade de Cuiabá, Curso de Odontologia, MT, Brasil \\ bUniversidade de Cuiabá, Programa de Pós-Graduação Stricto Sensu em Ciências Odontológicas Integradas, MT, Brasil \\ 'Universidade Anhaguera de São Paulo, Programa de Pós-Graduação em Farmácia, SP, Brasil \\ ${ }^{\mathrm{d} I n s t i t u t o ~ d e ~ E n s i n o ~ S u p e r i o r ~ d e ~ M a t o ~ G r o s s o, ~ C u r s o ~ d e ~ E n f e r m a g e m, ~ M T, ~ B r a s i l ~}$ \\ *E-mail: semenoff@uol.com.br \\ Recebido: 08 de julho de 2015; Aceito: 13 de novembro de 2015
}

\begin{abstract}
Resumo
Os pacientes cardiopatas parecem desconhecer os problemas relacionados com sua saúde bucal e as respectivas repercussões no sucesso de seu tratamento cirúrgico cardiovascular. O objetivo do estudo foi avaliar o conhecimento da saúde bucal entre pacientes cardiopatas internados, aguardando para serem submetidos às cirurgias cardíacas, com indicação de monitoramento na unidade de terapia intensiva (UTI). $\mathrm{O}$ estudo utilizou uma amostra composta por 62 pacientes de ambos os gêneros, com idade entre 33 e 87 anos. O instrumento de pesquisa consistiu de um questionário para registro de informações. Foram coletados dados referentes à: anamnese, condição socioeconômica, higiene bucal - escovação, aparatos de higiene, frequência de troca destes aparatos, frequência odontológica e presença de próteses dentais. Como critério de inclusão para o estudo, o indivíduo deveria ser cardiopata, estar internado e no aguardo de cirurgia cardíaca. Os dados foram organizados com auxílio do software IBM ${ }^{\circledR}$ SPSS ${ }^{\circledR}$ utilizando-se o teste estatístico Qui-Quadrado $(\mathrm{p}<0,05)$. A maioria dos pacientes era do sexo masculino (64,5\%), cor branca $(51,6 \%)$, idade média de $60,35+11,73$,não fumante $(91,9 \%)$ e não diabético $(75,8 \%)$. Para os hábitos de higiene, $87,1 \%$ escovavam os dentes duas ou três vezes ao dia, destes $98,4 \%$ utilizavam escova e creme dental, entretanto $69,4 \%$ não utilizam o fio dental. Do total, $56,5 \%$ pacientes estavam a mais de um ano sem ir ao dentista; destes, $33,9 \%$ tinham procurado este profissional em busca de exodontia. Quanto ao questionamento de como os pacientes consideravam sua saúde bucal, $37,1 \%, 43,5 \%$ e $19,4 \%$ disseram ser boa, regular e ruim, respectivamente. Do total, $48,4 \%$ dos pacientes perderam todos os dentes superiores e outros $30,6 \%$ todos os inferiores. Conclui-se que a maioria dos pacientes tem conhecimento sobre a importância de se escovar os dentes pelo menos duas vezes ao dia para a manutenção da saúde bucal, entretanto os indicadores avaliados demonstram que no geral o estado de saúde bucal é insatisfatório.
\end{abstract}

Palavras-chave: Higiene Bucal. Doenças Cardiovasculares. Unidades de Terapia Intensiva.

\begin{abstract}
Cardiac patients are unaware of the problems related to their oral health and its impact on the success of cardiovascular surgery. The aim of the study was to evaluate the knowledge of oral health among hospitalized cardiac patients waiting for cardiac surgery with monitoring indication in the intensive care unit. The study used a sample of 62 patients of both genders aged between 33 and 87 years. The research instruments consisted of a questionnaire for registration information. Data were collected regarding: medical history, socioeconomic status, oral hygiene - brushing, hygiene apparatus, and exchange frequency of these devices, dental attendance, and presence of dental prostheses. As inclusion criteria for the study, subjects could be cardiac, being hospitalized and awaiting heart surgery. Data were organized with the help of IBM SPSS software using the statistical test Chi-square ( $<<0.05)$. Most patients were male $(64.5 \%)$, white $(51.6 \%)$ aged $60.35+$ 11.73 , non-smoker (91.9\%) and non-diabetic (75.8\%). For hygiene, $87.1 \%$ brushed their teeth two or three times a day, of which $98.4 \%$ used toothbrush and toothpaste; however, $69.4 \%$ do not use the floss. A total of $56.5 \%$ patients was more than a year without going to the dentist; of these $33.9 \%$ had sought this professional looking for extraction. As for the question of how patients rated their oral health, 37.1\%, 43.5\%, and $19.4 \%$ considered it good, fair, and poor, respectively. A total of $48.4 \%$ of patients lost all upper teeth, and other $30.6 \%$ lost lower teeth. It is concluded that most patients know about the importance of brushing their teeth at least twice daily for the maintenance of oral health, though the appraised indicators show that the oral health status is unsatisfactory.
\end{abstract}

Keywords: Oral Hygiene. Cardiovascular Diseases. Intensive Care Units.

\section{Introdução}

$\mathrm{Na}$ cavidade bucal, encontra-se uma grande quantidade de microrganismos que vivem no corpo humano, incluindo os vírus, as bactérias e os fungos ${ }^{1,2}$ que formam o biofilme dental $^{3}$. Esta microbiota é capaz de interagir entre si e com o hospedeiro, determinando o que nos dias atuais chama-se de interação ecológica ${ }^{4}$.
Estudos recentes indicam que as doenças orais estão associadas com diversas patologias do corpo humano ${ }^{1,2}$, dentre elas as doenças cardíacas ${ }^{5}$. $\mathrm{Na}$ atualidade, entendemse as doenças orais em grande parte como um potencial de propagação de inflamação que, quando iniciado, é capaz de gerar mediadores inflamatórios como as interleucinas 1, 2 e 6 (IL1, IL2 e IL6), além do Fator de Necrose Tumoral - FNT, 
todos capazes de conduzir a modificações na microcirculação e na macrocirculação ${ }^{6,7}$.

As doenças cardiovasculares têm grande incidência na população. Trata-se de uma doença crônica inflamatória progressiva que se caracteriza pelo acúmulo de gordura no interior dos vasos, capazes de formar uma obstrução da circulação sanguínea ${ }^{8}$. Isso faz com que o indivíduo passe pela intervenção cirúrgica para tratamento da patologia, seguida da necessidade de acompanhamento na Unidade de Terapia Intensiva - UTI, o que qualifica a uma situação de alta complexidade ${ }^{9}$, principalmente pelo fato de que muitos pacientes cardíacos chegam a óbito. $\mathrm{Na}$ maioria dos casos, isso ocorre devido à infecção nosocomial adquirida dentro da UTI. Embora medidas em relação à higiene bucal destes pacientes tem se propagado, ainda necessita de atenção pelos riscos oferecidos ${ }^{10}$.

Os microrganismos Staphylococcus aureus, Pseudomonas aeruginosa, Staphylococcus epidermidis dentre outros, são bactérias importantes na composição da microbiota normal da pele e da mucosa humana e bastante relacionadas a situações de contaminação nosocomial. Na eventualidade de quebra da barreira inata cutânea natural e diminuição da imunidade, geralmente em pacientes internados, este fator pode ocasionar infecções oportunistas, e a cavidade bucal pode ser um dos albergues ${ }^{11}$.

Sabe-se que a má higienização bucal proporciona o surgimento de periodontite marginal que, por sua vez, relaciona-se com a piora de doenças sistêmicas como a endocardite bacteriana, aterosclerose e acidentes vasculares cerebrais. Além disso, microrganismos que colonizam as bolsas periodontais causam, com frequência, bacteremias no hospedeiro, em virtude do aumento da vascularização e da ulceração do epitélio gengival. Portanto, a presença de focos de infecção bucal pode conduzir a insucessos nos pósoperatórios de cirurgias cardíacas e sérias sequelas, inclusive com risco de morte no período de convalescência durante a internação na Unidade de Terapia Intensiva ${ }^{12,13}$.

Por outro lado, acredita-se que a prevenção dos processos infecciosos da cavidade oral é capaz de reduzir custos e evitar o número de óbitos decorrentes destes processos, portanto buscou-se compreender melhor a auto percepção do paciente cardiopata em aguardo de cirurgia cardíaca sobre sua saúde bucal. Frente ao exposto, a presente pesquisa objetivou avaliar o conhecimento sobre a saúde bucal entre pacientes internados, aguardando a cirurgia cardíaca com indicação de monitoramento na UTI.

\section{Material e Métodos}

O projeto deste estudo foi realizado em conformidade com a declaração de Helsinki, submetido e aprovado pelo comitê de ética em pesquisa da Universidade de Cuiabá Unic, 2012-053 e recebeu parecer favorável da Comissão de Pesquisa do Hospital Geral Universitário - HGU.

Os participantes da pesquisa foram informados sobre a natureza do trabalho e a necessidade de obtenção de uma autorização antes da realização de qualquer procedimento, conforme orientações contidas na Resolução 466/2012, do Conselho Nacional de Saúde para pesquisa envolvendo seres humanos.

Os pacientes que aceitaram participar da pesquisa assinavam o termo de consentimento esclarecido e um único pesquisador realizava as perguntas do questionário validado anteriormente em estudo piloto realizado seis meses antes com pacientes cardiopatas atendidos ambulatorialmente na Clínica de Odontologia da Universidade de Cuiabá - UNIC. O pesquisador/entrevistador oferecia as alternativas de resposta para cada uma das perguntas e preenchia o questionário com as respostas fornecidas pelos pacientes.

O questionário foi composto por anamnese, questões relacionadas às condições socioeconômicas e à higiene bucal, tais como: frequência de escovação, tipo de material utilizado para a higiene bucal, frequência de troca destes aparatos, frequência de ida ao dentista, perda de dentes, atendido pelo serviço público ou privado e presença de prótese dental.

Os dados foram codificados para todas as variáveis e categorias estudadas, tabulados no programa estatístico IBM ${ }^{\circledR}$ SPSS $\AA^{2} 20$ e submetidos ao teste de Qui Quadrado. O estudo teve uma significância de 5\%.

\section{Resultados e Discussão}

De acordo com o Quadro 1, referente à identificação geral dos pacientes conforme os dados sociodemográficos, verificouse que no total de 62 pacientes cardiopatas entrevistados, $40(64,5 \%)$ eram do sexo masculino e $22(35,5 \%)$ do sexo feminino, demonstrando diferença estatística ( $\mathrm{p}<0,031)$. Destes, $32(51,06 \%)$ declararam ser de cor branca e $30(48,4 \%)$ de cor negra, sem haver diferenças estatísticas entres estes ( $p>0,899)$. Do total de pacientes, 15 (24,2\%) eram diabéticos e 47 (75,8\%) não diabéticos, com diferença estatística $(\mathrm{p}<0,002)$. Entre os pacientes avaliados, $57(91,9 \%)$ diziam ser não fumantes e 5 $(8,1 \%)$ fumantes, havendo diferença estatística $(p<0,001)$. Em relação à classe social, 13 (21\%) pacientes eram da classe B, $33(53,2 \%)$ da classe C e $16(25,8 \%)$ pacientes na classe D-E. Não houve indivíduos da classe social $\mathrm{A}$ e a classe social $\mathrm{C}$ foi estatisticamente mais prevalente $(p<0,004)$ em relação às classes sociais B e D/E. A idade predominante foi de 60,35 anos com Desvio Padrão de 11,73, variando entre 33 e 87 anos. Com relação ao tempo de patologia, os pacientes relataram um tempo médio de 65,28 meses, sendo que o tempo mínimo descrito foi de um mês e o tempo máximo de 420 meses (Quadro 1).

Quadro 1: Identificação Geral dos Pacientes conforme os dados sociodemográficos

\begin{tabular}{|l|l|c|c|c|c|}
\hline \multicolumn{1}{|c|}{ Categoria } & Variáveis & Frequência & $\mathbf{\%}$ & & $\mathbf{p}$ \\
\hline \multirow{2}{*}{ Sexo } & Masculino & 40 & 64,5 & $\mathbf{A}$ & \multirow{2}{*}{$\mathrm{p}<0,031$} \\
\cline { 2 - 6 } & Feminino & 22 & 35,5 & $\mathbf{B}$ & \\
\hline \multirow{2}{*}{ Cor da Pele } & Branca & 32 & 51,6 & $\mathbf{A}$ & \multirow{2}{*}{$\mathrm{p}>0,899$} \\
\cline { 2 - 5 } & Negra & 30 & 48,4 & $\mathbf{A}$ & \\
\hline
\end{tabular}




\begin{tabular}{|c|c|c|c|c|c|}
\hline Categoria & Variáveis & Frequência & $\%$ & & p \\
\hline \multirow{2}{*}{ Diabetes } & Não & 47 & 75,8 & $\mathbf{A}$ & \multirow{2}{*}{$\mathrm{p}<0,002$} \\
\hline & Sim & 15 & 24,2 & B & \\
\hline \multirow{2}{*}{ Fumante } & Não & 57 & 91,9 & $\mathbf{A}$ & \multirow{2}{*}{$\mathrm{p}<0,001$} \\
\hline & Sim & 5 & 8,1 & B & \\
\hline \multirow{3}{*}{ Socioeconômico } & Classe B & 13 & 21 & $\mathbf{A}$ & \multirow{3}{*}{$\mathrm{p}<0,004$} \\
\hline & Classe C & 33 & 53,2 & B & \\
\hline & Classe D-E & 16 & 25,8 & $\mathbf{A}$ & \\
\hline \multirow{2}{*}{ Idade } & Mínima & Média & \multicolumn{2}{|c|}{ Máxima } & DP \\
\hline & 33 & 60,35 & & 87 & 11,73 \\
\hline \multirow{2}{*}{$\begin{array}{l}\text { Tempo de } \\
\text { Patologia } \\
\text { (Meses) }\end{array}$} & Mínima & Média & \multicolumn{2}{|c|}{ Máxima } & $\mathrm{DP}$ \\
\hline & 1 & 65,28 & & 420 & 85,24 \\
\hline
\end{tabular}

(\%) porcentagem; (p) nível de significância; A e B diferenças estatísticas nos grupos.

Fonte: Dados da pesquisa.

Com relação ao número de escovação diária, duas (3,2\%) pessoas responderam que não higienizavam a boca, seis
$(9,7 \%)$ relataram higienizar uma vez ao dia e $54(87,1 \%)$ higienizavam duas ou mais vezes ao dia $(\mathrm{p}<0,001)$. Sobre os produtos utilizados para a escovação, $60(96,8 \%)$ pacientes afirmaram usar a escova dental junto com o creme dental; dois $(3,2 \%)$ afirmaram não usar escova de dente e nem creme dental $(\mathrm{p}<0,001) ; 43(69,4 \%)$ pacientes não utilizam o fio dental e 19 utilizavam o fio dental $(p<0,001)$. Ao serem questionados sobre possuir ou não uma única escova dental para família, $60(96,8 \%)$ pacientes responderam que tinham escovas individuais e dois $(3,2 \%)$ não utilizam escova, nem individual nem comunitária em sua família $(\mathrm{p}<0,001)$. Em relação ao tempo de troca da escova dental, $33(53,2 \%)$ pacientes declararam trocar em menos de três meses, 21 $(33,9 \%)$ pacientes declararam trocar a escova dental entre três e seis meses e $8(12,9 \%)$ pacientes declararam que troca a escova dental em mais de seis meses, havendo diferença estatística entre os tempos de troca $(\mathrm{p}<0,001)$ (Quadro 2). Vale ressaltar que os pacientes que declararam não escovar seus dentes foram inseridos no grupo que dizem trocar suas escovas em mais de seis meses.

Quadro 2: Cuidados de higiene e condição da saúde bucal dos pacientes

\begin{tabular}{|c|c|c|c|c|c|}
\hline Categoria & Variável & $\mathbf{N}$ & $\%$ & & p \\
\hline \multirow{3}{*}{ Escovação } & Não escova & 2 & 3,2 & $\mathbf{A}$ & \multirow{3}{*}{$\mathrm{p}<0,001$} \\
\hline & Uma vez & 6 & 9,7 & $\mathbf{A}$ & \\
\hline & Duas vezes ou mais & 54 & 87,1 & B & \\
\hline \multirow{2}{*}{ Uso de escova e creme dental } & Sim & 60 & 96,8 & $\mathbf{A}$ & \multirow{2}{*}{$\mathrm{p}<0,001$} \\
\hline & Não & 2 & 3,2 & B & \\
\hline \multirow{2}{*}{ Uso de fio dental } & Sim & 19 & 30,6 & $\mathbf{A}$ & \multirow{2}{*}{$\mathrm{p}<0,001$} \\
\hline & Não & 43 & 69,4 & B & \\
\hline \multirow{2}{*}{ Escova individual } & Sim & 60 & 96,8 & $\mathbf{A}$ & \multirow{2}{*}{$\mathrm{p}<0,001$} \\
\hline & Não & 2 & 3,2 & $\mathbf{B}$ & \\
\hline \multirow{3}{*}{ Troca de escova } & Menos 3 meses & 33 & 53,2 & $\mathbf{A}$ & \multirow{3}{*}{$\mathrm{p}<0,001$} \\
\hline & Entre 3 e 6 meses & 21 & 33,9 & B & \\
\hline & Mais que 6 meses & 8 & 12,9 & C & \\
\hline \multirow{3}{*}{ Saúde bucal } & Boa & 23 & 37,1 & $\mathbf{A}$ & \multirow{3}{*}{$\mathrm{p}<0,054$} \\
\hline & Regular & 27 & 43,5 & $\mathbf{A}$ & \\
\hline & Ruim & 12 & 19,4 & B & \\
\hline \multirow{4}{*}{$\begin{array}{l}\text { Tempo da última consulta } \\
\text { odontológica }\end{array}$} & Menos 1 ano & 25 & 40,3 & $\mathbf{A}$ & \multirow{4}{*}{$\mathrm{p}<0,001$} \\
\hline & Entre 1 e 3 anos & 9 & 14,6 & B & \\
\hline & Mais que 3 anos & 24 & 38,7 & $\mathbf{A}$ & \\
\hline & Nunca consultou & 2 & 3,2 & B & \\
\hline \multirow{5}{*}{ Motivo de ida ao dentista } & Manutenção & 8 & 12,9 & $\mathbf{A}$ & \multirow{5}{*}{$\mathrm{p}<0,001$} \\
\hline & Dor & 4 & 6,5 & $\mathbf{A}$ & \\
\hline & Extração & 21 & 33,9 & B & \\
\hline & Tratamento dentário & 9 & 14,5 & $\mathbf{A}$ & \\
\hline & Outras causas & 18 & 29 & B & \\
\hline \multirow{4}{*}{ Atendimento odontológico } & Particular & 34 & 54,8 & $\mathbf{A}$ & \multirow{4}{*}{$\mathrm{p}>0,001$} \\
\hline & SUS & 22 & 35,5 & $\mathbf{A}$ & \\
\hline & Convênio & 3 & 4,8 & B & \\
\hline & ONG's & 3 & 4,8 & B & \\
\hline
\end{tabular}




\begin{tabular}{|c|c|c|c|c|c|}
\hline Categoria & Variável & $\mathbf{N}$ & $\%$ & & $\mathbf{p}$ \\
\hline \multirow{4}{*}{ Perda dentes Superiores } & Não & 4 & 6,5 & $\mathbf{A}$ & \multirow{4}{*}{$\mathrm{p}>0,001$} \\
\hline & 1 a 4 dentes & 15 & 24,2 & B & \\
\hline & 5 ou mais dentes & 13 & 21 & B & \\
\hline & Todos & 30 & 48,4 & $\mathbf{C}$ & \\
\hline \multirow{4}{*}{ Perda de Dentes Inferiores } & Não & 5 & 8,1 & $\mathbf{A}$ & \multirow{4}{*}{$\mathrm{p}>0,001$} \\
\hline & 1 a 4 dentes & 12 & 19,4 & B & \\
\hline & 5 ou mais dentes & 26 & 41,9 & $\mathrm{C}$ & \\
\hline & Todos & 19 & 30,6 & BC & \\
\hline \multirow{2}{*}{ Uso de Prótese } & Não & 23 & 37,1 & $\mathbf{A}$ & \multirow{2}{*}{$\mathrm{p}>0,001$} \\
\hline & Sim & 39 & 62,9 & $\mathbf{A}$ & \\
\hline
\end{tabular}

(\%) significa; (p) nível de significância; A, B e C diferenças estatísticas nos grupos.

Fonte: Dados da pesquisa.

Sobre como o paciente avalia sua saúde bucal, 23 (37,1\%) consideraram boa, $27(43,5 \%)$ consideraram regular e 12 $(19,4 \%)$ ruim, sem diferença estatística entre os pacientes que consideram sua saúde bucal boa e regular ( $>>0,05)$, que foram significativamente diferentes do grupo que considera ruim a saúde bucal $(\mathrm{p}<0,05)$. Em relação ao tempo da última consulta odontológica, $25(40,3 \%)$ responderam menos de um ano, nove $(14,6 \%)$ entre um e três anos e $24(38,7 \%)$ pacientes não foram ao dentista há mais de três anos. $\mathrm{O}$ mais interessante deste tema é que dois $(3,2 \%)$ pacientes nunca foram ao dentista, com diferença estatística com os demais $(\mathrm{p}<0,001)$. Sobre qual o motivo da última consulta, oito $(12,9 \%)$ responderam manutenção; quatro $(6,5 \%)$ relataram dor; $21(33,9 \%)$ para extração; nove $(14,5 \%)$ disseram ser tratamento dentário; e 18(29\%) indicaram outras causas. Não houve diferenças estatísticas entre os pacientes que relataram atendimento odontológico decorrido de manutenção, dor e tratamento dentário $(\mathrm{p}>0,05)$; porém estes diferiram dos pacientes que se consultaram por necessidade de exodontia $(\mathrm{p}<0,001)$ (Quadro 2).

Em relação ao atendimento odontológico particular, conveniado ou público, $34(54,8 \%)$ pacientes foram atendidos em clínicas particulares; 22 (35,5\%) pelo Sistema Único de Saúde; três pacientes $(4,8 \%)$ pelo plano de saúde e outros três pacientes $(4,8 \%)$ em Organizações não Governamentais - ONG. Não houve diferenças estatísticas entre os pacientes atendidos em clínicas particulares e pelo Sistema Único de Saúde, entretanto ambos se diferiram daqueles atendidos pelos planos de saúde e ONG ( $\mathrm{p}<0,001)$ (Quadro 2).

Em relação à perda dos dentes superiores, quatro $(6,5 \%)$ pacientes não perderam dentes $15(24,2 \%)$ perderam entre 1 e 4 dentes; 13 (21\%) cinco ou mais dentes; e $30(48,4 \%)$ pacientes perderam todos os dentes superiores, com diferença estatística entre os pacientes que possuíam todos os dentes e os que sofreram exodontia de todos os dentes superiores $(p<0,001)$, sendo que ambos se diferiram daqueles que perderam menos dentes $(p<0,001)$. Em relação aos dentes inferiores, cinco $(8,1 \%)$ pacientes não perderam nenhum elemento dentário; $12(19,4 \%)$ perderam de um a quatro dentes; 26 (41,9\%) pacientes perderam mais de cinco dentes; 19(30,6\%) pacientes perderam todos os dentes inferiores, não havendo diferença estatística entre os pacientes que possuíam todos os dentes inferiores e os demais pacientes $(\mathrm{p}<0,001)$. Em relação aos usos de próteses, 23(37,1\%) pessoas não utilizavam próteses totais e 39 pessoas utilizam pelo menos uma prótese total $(55,6)$ (Quadro 2). Vale ressaltar que, destes pacientes, seis haviam perdido todos os elementos dentários superiores e inferiores.

Os resultados demonstraram que os pacientes eram na maioria do sexo masculino e com idade média de 60,35 anos e portadores da doença por 65 meses em média, ou seja, conhecem e convivem com a doença há algum tempo. Fatores de risco como o uso do tabaco e a presença de diabetes foram fatores poucos presentes, entretanto observa-se que os doentes se encontravam em momentos antes da cirurgia e cientes que adentrariam na UTI.

$\mathrm{Na}$ atualidade, sabe-se que a classe social baixa aumenta os riscos de doença periodonta $1^{12}$ e cárie ${ }^{13}$. Neste estudo, a maioria dos pacientes era da classe $\mathrm{C}$, portanto compatível com a presença das doenças citadas. Outro fator de destaque é que pacientes neste estrato tiveram pouco acesso à saúde e a informação, o que ocasiona dificuldades, além da desmotivação em relação aos autocuidados bucais. Estes fatores conduzem os indivíduos a viverem longe do dentista, conforme mostrado no estudo ${ }^{14}$. Diante deste quadro, ações a respeito das informações sobre a saúde bucal devem ser estimuladas, com objetivo de diminuir riscos diante da passagem do paciente pela UTI.

A maioria dos pacientes relata que escova os dentes mais de duas vezes ao dia e troca as escovas em períodos adequados, contudo sabe-se que, de forma geral, os pacientes cardiopatas, assim como portadores de outros processos patológicos complexos, demonstram pobre higiene bucal e desmotivação ${ }^{15,16}$, 0 que ocasiona pioras nos indicadores de saúde bucal, como a presença de gengivite, periodontite e perda de dentes. 
Alguns dados como a não utilização adequada do fio dental ${ }^{13}$; percepção da saúde bucal insatisfatória e relatos de que $40 \%$ dos pacientes não visitam o dentista há pelo menos três anos, demonstram que, embora convivam por um tempo razoável com a doença, desconhecem os riscos da relação entre a presença de infecção bucal e cardiopatia ${ }^{5}$. Neste sentido, ações simples com objetivo de esclarecer e motivar os pacientes tornam-se importantes ${ }^{13}$.

O histórico de perdas de dentes evidencia os riscos de inflamação através do foco de infecção bucal ${ }^{5}$. Em estudo longitudinal, percebe-se que a perda de dentes se relaciona com o aparecimento de doenças cardíacas, além da presença de óbitos ${ }^{17}$. Em relação ao estudo, a maioria dos pacientes utilizavam próteses totais e perderam mais de cinco dentes, o que são dois indicadores negativos de saúde bucal e do coração ${ }^{7}$. De cada cinco pessoas avaliadas, três não usavam o fio dental, constatando a falta de conhecimento ou orientação sobre os benefícios do uso do fio dental para a saúde bucal ${ }^{13}$.

Os resultados do trabalho evidenciam que medidas devem ser realizadas para evitar complicações com infecções orais. Lembra-se que há na atualidade grandes complicações somente com a presença de infecção bucal em relação a diversas doenças no corpo, inclusive com risco de morte ${ }^{18,19}$. Ao estabelecer tratamento de alta complexidade como a UTI, cuidados devem ser ainda mais atentos. Objetivamente, medidas pré-operatórias em relação à saúde bucal reduzem custos $^{20,21} \mathrm{e}$ devem ser amplamente inseridas em pacientes que sofrerão o estresse de uma cirurgia cardíaca e passarão pela UTI.

\section{Conclusão}

Conclui-se que a maioria dos pacientes tem conhecimento sobre a importância de se escovar os dentes pelo menos duas vezes ao dia para a manutenção da saúde bucal, entretanto os indicadores avaliados demonstraram cuidados mínimos com a saúde bucal de pacientes que passarão pela UTI.

\section{Agradecimentos}

A Fundação de Amparo a Pesquisa do Estado de Mato Grosso - FAPEMAT.

\section{Referências}

1. Santos PSS, Mariano M, Kallas MS, Vilela MCN. Impact of tongue biofilm removal on mechanically ventilated patients. Rev Bras Ter Intensiva 2013;25(1):44-8.

2. Slots J, Slots H. Bacterial and viral pathogens in saliva: disease relationship and infectious risk. Periodontol 2000 2011;55(1):48-69.

3. Haffajee AD, Socransky SS. Introduction to microbial aspects of periodontal biofilm communities, development and treatment. Periodontol 2000 2006;42:7-12.

4. Socransky SS, Haffajee AD. Periodontal microbial ecology. Periodontol 2000 2005;38:135-87.
5. Kholy KE, Genco RJ, Van Dyke TE. Oral infections and cardiovascular disease. Trends Endocrinol Metab 2015;26(6):315-321.

6. Libby P. Role of inflammation in atherosclerosis associated with rheumatoid arthritis. Am J Med 2008;121(10):S21-31.

7. Kozarov EV. Human atherosclerotic plaque contains viable invasive Actinobacillus actinomycetemcomitans and Porphyromonas gingivalis. Arterioscler Thromb Vasc Biol 2005;25(3):17-18.

8. Lusis AJ. Atherosclerosis. Nature 2000;407(6801):233-41.

9. Celakovsky P, Kalfert D, Smatanova K, Tucek L, Cermakova $\mathrm{E}$, Mejzlik J, et al. Bacteriology of deep neck infections: analysis of 634 patients. Aust Dent J 2015;60(2):212-5.

10. Araújo RJG, Oliveira LCG, Hanna LMO, Corrêa AM, Carvalho LHV, Alvares NCF. Análise de percepções e ações de cuidados bucais realizados por equipes de enfermagem em unidades de tratamento intensivo. Rev Bras Ter Intensiva 2009;21(1):38-44.

11. Segers P, Speekenbrink RG, Ubbink DT, van Ogtrop ML, de Mol BA. Prevention of nosocomial infection in cardiac surgery by decontamination of the nasopharynx and oropharynx with chlorhexidine gluconate. A randomized controlled trial. JAMA 2006;296:2460-6.

12. Susin C, Haas AN, Valle PM, Oppermann RV, Albandar JM. Prevalence and risk indicators for chronic periodontitis in adolescents and young adults in South Brazil. J Clin Periodontol 2011;38:326-33. doi: 10.1111/j.1600051X.2011.01699.x.

13. Veiga NJ, Pereira CM, Ferreira PC, Correia IJ. Prevalence of dental caries and fissure sealants in a Portuguese sample of adolescents. PLoS ONE 2015;10(3):e0121299. doi: 10.1371/ journal. pone.0121299.

14. Paula JS, Sarracini KLM, Meneghim MC, Pereira AC, Ortega EMM, Martins NS, et al. Longitudinal evaluation of the impacto of dental caries treatment on oral healthrelated quality of life among schoolchildren. Eur Oral Sci 2015;123:173-8.

15. Ericsson JS, Ostberg A-L, Wennstrom JL, Abrahamsson $\mathrm{KH}$. Oral health-related perceptions, attitudes, and behavior in relation to oral hygiene conditions in an adolescent population. Eur J Oral Sci 2012;120:335-41.

16. Munro CL. Oral health: something to smile about! Am J Crit Care 2014;23(4):282-8.

17. Liljestrand JM, Havulinna AS, Paju S, Männistö S, Salomaa V, Pussinen PJ. Missing teeth predict incident cardiovascular events, diabetes, and death. J Dent Res 2015;94(8)1055-62.

18. Beikler T, Flemming TF. Oral biofilm-associated diseases: trends and implications for quality of life, systemic health and expenditures. Periodontol 2000 2011;55(1):87-103.

19. Socransky SS, Haffajee AD. Dental biofilms: difficult therapeutic targets. Periodontol 2000 2002;28:12-55.

20. Bhaskar V, McGraw KA, Divaris K. The importance of preventive dental visits from a young age: systematic review and current perspectives. Clin Cosm Invest Dent 2014;20(8):21-7.

21. Morais TMN, Silva A, Avi ALRO, Souza PHR, Knobel E, Camargo LFA. A importância da atuação odontológica em pacientes internados em unidade de terapia intensiva. Rev Bras Ter Intensiva 2006;18(4):412-7. 